\title{
The Characterization of Linear Polyethylene SRM's 1482, 1483, and 1484. IV. Limiting Viscosity Numbers by Capillary Viscometry
}

\author{
Herman L. Wagner and Peter H. Verdier \\ Institute for Materials Research, National Bureau of Standards, Washington, D.C. 20234
}

(October 19, 1978)

\begin{abstract}
Linear polyethylene Standard Reference Materials SRM 1482, 1483, and 1484 are certified for limiting viscosity number in $1,2,4$-trichlorobenzene and 1-chloronaphthalene at $130{ }^{\circ} \mathrm{C}$. In this paper the experimental procedures employed for the determination of limiting viscosity numbers for these materials by capillary viscometry are described, and the techniques used to analyze the data and to estimate limits of systematic error are discussed.
\end{abstract}

Key words: Capillary viscometry; fraction; limiting viscosity number; narrow molecular weight distribution; number-average molecular weight; polyethylene; Standard Reference Material; weight-average molecular weight.

\section{Introduction}

Standard Reference Materials 1482, 1483, and 1484 are linear polyethylenes with relatively narrow distributions in molecular weight, which are issued by the National Bureau of Standards. Their general characteristics are described in the first paper of this series [1]. ${ }^{1}$ In the present paper, we describe the determination of their certificate values of limiting viscosity number at $130^{\circ} \mathrm{C}$ in 1,2,4-trichlorobenzene (TCB) and in 1-chloronaphthalene ( $\mathrm{ICN})$. The determination of limiting viscosity number by capillary viscometry is relatively simple. Although the relationship between limiting viscosity number and molecular weight remains analytically intractable, it has been well established empirically for linear polyethylene in the solvents and at the temperature cited above [2].

\section{Experimental}

Viscosity measurements were made with a Cannon-Ubbelohde filter stick viscometer (Cannon Instrument Co., State College, Pa., ${ }^{2}$ size 75 ) immersed in a constant-temperature bath. Flow times in the viscometer were measured with a Hewlett-Packard Autoviscometer, Model 5901B.

Flow times were measured in both solvents for solutions whose concentrations ranged from 2 to $9 \mathrm{~g} / \mathrm{L}$ for SRM 1482 , 1 to $5 \mathrm{~g} / \mathrm{L}$ for SRM 1483 , and 0.3 to $2 \mathrm{~g} / \mathrm{L}$ for SRM 1484 . All solutions were made up directly by weight, without employing successive dilution techniques. Concentrations were calculated using values of solvent density and partial specific volume determined pycnometrically in this laboratory. The ICN was obtained from commercial material by distillation at reduced pressure after removal of residual naphthalene by sublimation, also at reduced pressure. Several sources of TCB were used, without apparent effect on

${ }^{1}$ Figures in brackets indicate literature references at the end of this paper.

${ }^{2}$ Certain commercial equipment, instruments, or materials are identified in this paper in order to adequately specify the experimental procedure. In no case does such identification imply recommendation or endorsement by the National Bureau of Standards, nor does it imply that the material or equipment identified is necessarily the best available for the purpose. the results obtained. From 0.05 to 0.1 percent of the antioxidant 2,6-di-tert-butyl-4-methylphenol was added to all solvents. The polyethylenes were dissolved by heating the solvent to $130-140{ }^{\circ} \mathrm{C}$ with occasional stirring, and the solutions were then tranferred to the viscometer through a sintered glass frit, as described in ASTM D 1601-61. ${ }^{3}$ A few minutes were allowed for thermal equilibration, then flow times were measured repeatedly until a value stable to $10 \mathrm{~ms}$ or so was obtained. The flow times measured in this way ranged from 70 to $100 \mathrm{~s}$ in TCB, and from 100 to $150 \mathrm{~s}$ in lCN.

\section{Results}

The solution viscosity $\eta(c)$ may be expanded as a power series in solution concentration $c$ :

$$
\eta(c)=P^{\prime}+Q^{\prime} c+R^{\prime} c^{2}+\cdots .
$$

The viscosity number, defined as $[\eta(c)-\eta(0)] /[\eta(0) c]$, is then:

$$
[\eta(c)-\eta(0)] /[\eta(0) c]=Q^{\prime} / P^{\prime}+\left(R^{\prime} / P^{\prime}\right) c+\cdots .
$$

The limiting viscosity number, for which we shall employ the symbol $\Lambda$ in this paper, ${ }^{4}$ is just the zero-concentration limit of the viscosity number [3], and is given by the ratio $Q^{\prime} / P^{\prime}$ of the coefficients in eq (1). The zero-concentration derivative of viscosity number with respect to concentration is usually expressed as $\Lambda^{2} k^{\prime}$, where $k^{\prime}$ is called the Huggins coefficient [3] and is given in terms of the coefficients in eq (1) by $P^{\prime} R^{\prime} / Q^{\prime 2}$

For a properly designed capillary viscometer, the solution viscosity is almost proportional to the product of solution density and measured flow time [4]. The departure from

\footnotetext{
${ }^{3}$ Available from the American Society for Testing and Materials, 1916 Race Street, Philadelphia, Pa. 19103.

The symbols LVN and $[\eta]$ are often used to denote this quantity; here we prefer a symbol which is not an acronym and is free of punctuation marks.
} 
proportionality is due to a combination of kinetic energy effects and hydrodynamic effects at the ends of the capillary. For the viscometer design and Reynolds numbers used in this work, the relationship between viscosity and flow time is given approximately [5] by:

$$
\eta / \rho=C t_{m}-E t_{m}^{-2}
$$

where $\rho$ is the density of the liquid, $t_{m}$ is the measured flow time through the capillary, and $C$ and $E$ are constants of the viscometer, determined experimentally. Since only ratios of viscosities are needed for the determination of limiting viscosity number and Huggins coefficient, it is convenient to introduce a "corrected" flow time $t$, defined by:

$$
t=t_{m}\left(1-K t_{m}^{-3}\right)
$$

with $K=E / C$. In terms of $t$, eq (2) becomes $\eta / \rho=C t$, and using eq (1), we can write the product $\rho t$ as a polynomial in concentration:

$$
\rho(c) t(c)=P+Q c+R c^{2}+\cdots,
$$

where $P, Q$, and $R$ are equal to the corresponding primed quantities in eq (1) divided by the viscometer constant $C$, and we have $\Lambda=Q / P, k^{\prime}=P R / Q^{2}$.

In the remainder of this section, we discuss first the method used to obtain the constant $K$ for the viscometer employed in this work, then the procedures used to obtain limiting viscosity numbers and Huggins coefficients for SRM's 1482, 1483, and 1484 from the "corrected" flow times, and finally the procedures employed to estimate the effect of shear rate upon the results.

\subsection{Correction for Kinetic Energy and End Effects}

The correction factor $K$ in eq (3) was determined by measuring flow times for two certified viscosity standards (Cannon Instrument Co., State College, Pa.), of known viscosity and density. One (N4) of these was chosen to give a flow time close to that obtained with 1,2,4-trichlorobenzene, the less viscous of the solvents in which limiting viscosity numbers are reported. The other (S3) was chosen to have a very long flow time, giving a "correction" $\mathrm{Kt}_{m}^{-3}$ of about $3 \times 10^{-5}$. Six measurements were made with $\mathrm{N} 4$ and four with S3. A measurement consisted of charging the viscometer with liquid and measuring flow time repeatedly until a constant flow time was reached. The resulting mean flow times were used in eq (2) to determine $K$. Letting the subscripts $N$ and $S$ refer to measurements made on $\mathrm{N} 4$ and S3, respectively, we write eq (2) for each mean flow time and solve the resulting pair of equations for $E / C$, obtaining:

$$
K=E / C=\left(r t_{N}-t_{S}\right) /\left(r t_{N}^{-2}-t_{S}^{-2}\right),
$$

where $r=\eta_{S} \rho_{N} /\left(\eta_{N} \rho_{S}\right)$.

The standard deviations of the mean flow times amounted to about 0.01 percent for both liquids. Using Student $t$ factors [6] for 5 and 3 degrees of freedom, we obtain 95 percent confidence limits of 0.02 percent and 0.03 percent for flow time measurements on N4 and S3, respectively. The effects of these uncertainties upon the value of $K$, and ultimately upon the values of $\Lambda$, are discussed in section 4.3 .

\subsection{Limiting Viscosity Numbers}

Limiting viscosity numbers were determined, for each SRM and each solvent, by measuring flow times for solvent and for solutions of several different concentrations. The measured flow times were first converted to "corrected" flow times, using eq (3). The corrected flow times were then multiplied by solution densities calculated from measured values of solvent density and partial specific volume. In preliminary studies, it was found that for concentrations $c$ such that the ratios $\rho(c) t(c) /[\rho(0) t(0)]$ were less than 1.4 , plots of $\{\rho(c) t(c) /[\rho(0) t(0)]-1\} / c$ versus $c$ appeared linear, in accordance with eq (4). The certification measurements were therefore carried out in this concentration range, and the products $\rho(c) t(c)$ were fitted by least squares to secondorder polynomials in solution concentration $c$, as shown in eq (4). The limiting viscosity numbers $\Lambda$ and Huggins coefficients $k^{\prime}$ were then obtained as the ratios $Q / P$ and $P R / Q^{2}$ of the coefficients in eq (4).

In principle, the flow time for a given viscometer, solvent, and temperature ought to be constant in time. In practice, however, solvent flow times appeared to be materially more reproducible within a single day than over longer periods. Standard deviations of solvent flow times measured within a single day were typically about $16 \mathrm{~ms}$; standard deviations of flow times measured on different days were about twice this value. Measurements on each SRM and in each solvent were therefore grouped into subsets, each consisting of one or two values of solvent flow time and values of flow time for several solutions. All the measurements in each subset were made on the same day. The flow times in each subset were fitted separately by least squares to eq (4). For each SRM and each solvent, several subsets of data were obtained, leading to several estimates of $\Lambda$ and $k^{\prime}$. In some cases, the subsets were far from equivalent, some subsets containing twice as many measurements as others. The structure of the subsets and data analysis techniques employed are discussed separately for each of the three SRM's in the following subsections.

\section{a. SRM 1482}

Five subsets of measurements on SRM 1482 were made in each solvent. Each subset consists of from six to twelve flow time measurements, including solvent flow times. The solutions had nominal concentrations of $2,3,4.5,7$, and $9 \mathrm{~g} / \mathrm{L}$. Some nominal concentrations were omitted from some of the subsets; other subsets contain more than one solution of the same nominal concentration. Consequently, the estimates of $\Lambda$ and $k^{\prime}$ obtained from the separate subsets differ appreciably in precision. The final estimates of $\Lambda$ and $k^{\prime}$ in each solvent were therefore taken as weighted averages of the estimates from the individual subsets, obtained as described in the following paragraph.

Let the number of flow time measurements (of both solvent and solutions) in the $i$ th subset be $N_{i}$. The least squares analysis for the $i$ th subset yields an estimate $\Lambda_{i}$ of limiting viscosity number, together with an estimate $v_{i}(t)$ of the variance in flow time inferred from the mean-square differences between observed and calculated (from eq (4)) flow times, and an estimate $v\left(\Lambda_{i}\right)$ of the variance in $\Lambda_{i}$. Each estimate $v\left(\Lambda_{i}\right)$ is the product of $v_{i}(t)$ and a factor obtained from the covariance matrix for the $i$ th subset by standard 
techniques [6]. The scatter in the values $v\left(\Lambda_{i}\right)$ therefore reflects both the differences between the covariance matrices for the subsets and the scatter in the individual estimates $v_{i}(t)$. We now make the assumption that all the $v_{i}(t)$ for a given solvent are estimates of the same quantity $v(t)$. Since three parameters are fitted in each subset analysis, the number $F_{i}$ of degrees of freedom associated with $v_{i}(t)$ is just $N_{i}-3$. Our best estimate of $v(t)$ is given by the average of the $v_{i}(t)$ over all the subsets, weighted by the $F_{i}$, i.e.,

$$
v(t)=F^{-1} \sum_{i} F_{i} v_{i}(t)
$$

where $F=\sum_{i} F_{i}$ and the summations are taken over all the subsets for a given solvent. We can then obtain improved estimates $v^{\prime}\left(\Lambda_{i}\right)$ of the variance in $\Lambda_{i}$, which are free of the scatter in the individual estimates $v_{i}(t)$, as

$$
v^{\prime}\left(\Lambda_{i}\right)=v\left(\Lambda_{i}\right) v(t) / v_{i}(t) .
$$

Finally, we obtain our overall estimate of $\Lambda$ as the average of the $\Lambda_{i}$, weighted inversely by the $v^{\prime}\left(\Lambda_{i}\right)$, i.e.,

$$
\Lambda=\left[\sum_{i} v^{\prime}\left(\Lambda_{i}\right)^{-1}\right]^{-1} \sum_{i} v^{\prime}\left(\Lambda_{i}\right)^{-1} \Lambda_{i},
$$

with variance $v(\Lambda)$ given by

$$
v(\Lambda)=\left[\sum_{i} v^{\prime}\left(\Lambda_{i}\right)^{-1}\right]^{-1},
$$

with $F$ degrees of freedom. Finally, the standard deviation $s(\Lambda)$ in $\Lambda$ is obtained as $s(\Lambda)=[v(\Lambda)]^{1 / 2}$. Overall estimates of $k^{\prime}$ and $s\left(k^{\prime}\right)$ are obtained by an exactly analogous procedure. The results are given in table 1 .

\section{b. SRM 1483}

Five subsets of measurements on SRM 1483 were made in TCB. Each subset contains five solution flow times, for solutions with nominal concentrations of $1,2,3,4$, and $5 \mathrm{~g} / \mathrm{L}$, and two solvent flow times. Since the subsets are essentially equivalent, we obtain our final values for $\Lambda$ and $k^{\prime}$ as the unweighted averages of the estimates from the individual subsets. Three parameters are determined for each subset, so four degrees of freedom are associated with the estimates of the variance in $\Lambda$ and $k^{\prime}$ from each subset. Additional estimates of the variance in $\Lambda$ and $k^{\prime}$, also associated with four degrees of freedom, are obtained from the set of five individual subset estimates of $\Lambda$ and $k^{\prime}$. Our final estimates of the variance in the subset values of $\Lambda$ and $k^{\prime}$ are then formed as the unweighted average of the six individual estimates, one from each subset analysis and one from the set of subset values of $\Lambda$ and $k^{\prime}$, and are associated with a total of 24 degrees of freedom. The estimated variances $v\left(\Lambda_{m}\right)$ and $v\left(k_{m}{ }^{\prime}\right)$ of the final mean values of $\Lambda$ and $k^{\prime}$ are then of course just $1 / 5$ the estimated variances $v(\Lambda)$ and $v\left(k^{\prime}\right)$ of the individual values.

The estimated variance $v(t)$ and standard deviation $s(t)$ in flow time are not used directly in the calculation of $\Lambda, k^{\prime}$ and their variances. They may be obtained by observing that for equivalent subsets, the ratio $v(t) / v(\Lambda)$ of the variance of a single measurement of flow time to the variance of a single subset determination of $\Lambda$ is constant. Choosing the quantity $\sum_{i} v_{i}(t) / \sum_{i} v\left(\Lambda_{i}\right)$ to estimate this ratio, we have

$$
\begin{aligned}
v(t) & =s^{2}(t)=v(\Lambda) \sum_{i} v_{i}(t) / \sum_{i} v\left(\Lambda_{i}\right) \\
& =5 v\left(\Lambda_{m}\right) \sum_{i} v_{i}(t) / \sum_{i} v\left(\Lambda_{i}\right),
\end{aligned}
$$

where $v\left(\Lambda_{i}\right)$ and $v_{i}(t)$ are the estimated variances in $\Lambda$ and in $t$, respectively, obtained from the $i$ th subset analysis.

Four subsets of measurements on SRM 1483 were made in $1 \mathrm{CN}$. Three of these were identical in structure with the subsets in TCB. The fourth subset differed from the other three only in the omission of one of the solvent flow time measurements. We shall refer to this subset as the six-point subset, and to the others as the seven-point subsets. We first obtain estimates of $\Lambda, k^{\prime}, v(\Lambda), v\left(k^{\prime}\right)$, and $v(t)$ from the three equivalent seven-point subsets, in a way analogous to the method employed for the measurements on SRM 1483 in TCB. The variances so obtained are associated with 14 degrees of freedom, four from each subset and two from the subset-to-subset variation. We then combine these estimates with those from the six-point subset, associated with three degrees of freedom, in the way described in section $3.2 \mathrm{a}$, obtaining the final values given in table 1 , with a total of 17 degrees of freedom.

\section{c. SRM 1484}

\begin{tabular}{|c|c|c|c|c|c|c|c|}
\hline Solvent & Material & $s(t)$ seconds & $\begin{array}{c}\text { Limiting viscos- } \\
\text { ity number, } \Lambda \text {, } \\
\mathrm{mL} / \mathrm{g}\end{array}$ & $\begin{array}{l}\text { Standard de- } \\
\text { viation in } \Lambda, \\
\mathrm{mL} / \mathrm{g}\end{array}$ & $\begin{array}{l}\text { Huggins coeffi- } \\
\text { cient, } k^{\prime}\end{array}$ & $\begin{array}{l}\text { Standard devia- } \\
\text { tion in } k^{\prime}\end{array}$ & $\begin{array}{l}\text { Number of de- } \\
\text { grees of free- } \\
\text { dom }\end{array}$ \\
\hline \multirow[t]{3}{*}{ TCB } & SRM 1482 & 0.052 & 40.15 & 0.13 & 0.399 & 0.011 & 28 \\
\hline & SRM 1483 & .056 & 79.40 & .31 & .419 & .013 & 24 \\
\hline & SRM 1484 & .039 & 197.93 & .60 & .462 & .010 & 22 \\
\hline \multirow[t]{3}{*}{$1 \mathrm{CN}$} & SRM 1482 & .064 & 36.36 & .11 & .446 & .011 & 24 \\
\hline & SRM 1483 & .064 & 70.56 & .28 & .461 & .016 & 17 \\
\hline & SRM 1484 & .064 & 169.38 & .60 & .526 & .014 & 22 \\
\hline
\end{tabular}

Five subsets of measurements on SRM 1484 were made in each solvent. Each subset contains one or two flow times for

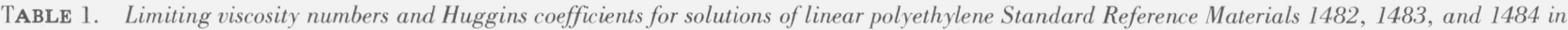
1,2,4-trichlorobenzene (TCB) and 1-chloronaphthalene (1CN) at $130^{\circ} \mathrm{C}$, obtained by capillary viscometry standard deviations $s(t)$ in viscometer flow times are also shown, as an indication of the overall precision of the measurements. 
solvent, flow times for two solutions with nominal concentrations of $0.6 \mathrm{~g} / \mathrm{L}$, and one flow time each for solutions with nominal concentrations of $0.4,0.8,1.2$, and $1.8 \mathrm{~g} / \mathrm{L}$. Since the number of solvent flow times is not the same in every subset, the subsets are not equivalent. They were therefore analyzed by the same technique employed for SRM 1482 and described in section $3.2 \mathrm{a}$. The results are shown in table 1 .

\subsection{Shear Rate Dependence}

At low rates of shear, the viscosity of a solution of flexible macromolecules is independent of shear rate. At higher rates of shear, however, solution viscosity decreases with increasing shear rate. The possibility of dependence of our.measured viscosities upon shear rate was investigated by a series of comparison measurements in two capillary viscometers with different shear rates. Since shear-rate dependence increases with increasing molecular weight, measurements were made on solutions of SRM 1484, the highest in molecular weight of the three SRM's.

The maximum shear rate in a capillary viscometer is given [7] in terms of the flow time $t$ and the bulb volume $V$ and capillary radius $a$ of the viscometer by the expression $4 V /\left(\pi a^{3} t\right)$. For the viscometer employed for the measurements described in section 3.2, hereafter referred to as the reference viscometer, this gives a maximum shear rate of about $3 \times 10^{3} \mathrm{~s}^{-1}$ for measurements in TCB at $130{ }^{\circ} \mathrm{C}$. The viscometer employed for comparison had a maximum shear rate of about $6 \times 10^{3} \mathrm{~s}^{-1}$ for the same solvent and temperature.

Flow times for solvent and for a solution of SRM 1484 with a nominal concentration $c$ of $1 \mathrm{~g} / \mathrm{L}$ were measured in both viscometers. The kinetic energy and end effect correction factor $K$, determined as described in section 3.1, was applied to the flow times measured with the reference viscometer. The corresponding correction for the comparison viscometer was found to be negligible from the manufacturer's calibration measurements. The ratios $t(c) / t(0)$ of solution flow time to solvent flow time were 1.219 for the reference viscometer and 1.221 for the comparison viscometer. The difference between them is consistent with our estimated precision of 0.001 in the ratio, and is in the opposite direction from the expected effect of shear-rate dependence, which would lead to a smaller ratio at higher shear rates. Thus we are unable to detect any shear-rate dependence in our results.

\section{Systematic Errors}

We now list the likeliest sources of systematic error in the estimates of limiting viscosity number described in the preceding section, and attempt to set upper limits on their magnitudes. Individual sources of error are discussed in the following subsections; the resulting error-limit estimates are summarized in table 2 , expressed as percent errors applied to $\Lambda$.

In this work, $\Lambda$ is calculated as the ratio $Q / P$ of coefficients in eq (4), which are obtained by fitting the product $\rho(c) t(c)$ to a second-order polynomial in solution concentration $c$ by least squares. Here $\rho(c)$ is solution density and $t(c)$ is related to the measured flow time $t_{m}(c)$ in the viscometer by eq (3). The correction factor $K$ in eq (3) is obtained as described in section 3.1 . The concentration is given by the product $w \rho(c)$, where $w$ is the weight fraction of solute in the solution, and $\rho(c)$ is given in terms of the solvent density $\rho(0)$ and the partial specific volume $\bar{v}$ by:

$$
\rho(c)=\rho(0) /[1-w(1-\rho(0) \bar{v}] .
$$

Systematic errors in $\rho(0), \bar{v}, K, w$, and $t_{m}(c)$ will therefore affect the calculated values of $\Lambda$ directly. The effects of errors in the first three of these quantities can be exhibited explicitly. For this purpose, we first write, using eq (4):

$$
\Lambda=\mathscr{L}\{[\rho(c) t(c)-\rho(0) t(0)] /[\rho(0) t(0) c]\},
$$

where we use the symbol $\mathscr{L}\{\}$ to mean the zero-concentration limit of the quantity within the curly brackets. We then use eq (3) and (9) to re-express the limit in eq (10) in terms of a limit which involves only the directly measured quantities $w$ and $t_{m}(c)$. After some rearrangement, and making use of the fact that the limit of a product is the product of the limits of the factors when all the limits involved exist, we find:

$$
\begin{aligned}
\Lambda= & \rho(0)^{-1}\left[\left[1+2 K t_{m}(0)^{-3}\right] /\left[1-K t_{m}(0)^{-3}\right]^{-1}\right. \\
& \left.\times \mathscr{L}\left\{\left[t_{m}(c)-t_{m}(0)\right] /\left[w t_{m}(0)\right]\right\}+1\right]-\bar{v}
\end{aligned}
$$

from which the effects of errors in $\rho(0), K$, and $\bar{v}$ upon $\Lambda$ may

\begin{tabular}{|c|c|c|c|c|c|c|c|}
\hline \multicolumn{2}{|c|}{ Source of error } & \multicolumn{6}{|c|}{ Error in limiting viscosity number, percent } \\
\hline & \multirow{2}{*}{$\begin{array}{l}\text { Solvent } \\
\text { Material }\end{array}$} & \multicolumn{3}{|c|}{$\begin{array}{l}\text { 1,2,4-trichloroben- } \\
\text { zene }\end{array}$} & \multicolumn{3}{|c|}{ 1-chloronaphthalene } \\
\hline & & $\begin{array}{l}\text { SRM } \\
1482\end{array}$ & $\begin{array}{l}\text { SRM } \\
1483\end{array}$ & $\begin{array}{l}\mathrm{SRM} \\
1484\end{array}$ & $\begin{array}{l}\text { SRM } \\
1482\end{array}$ & $\begin{array}{l}\text { SRM } \\
1483\end{array}$ & $\begin{array}{l}\text { SRM } \\
1484\end{array}$ \\
\hline \multicolumn{2}{|l|}{ Solvent density } & 0.20 & 0.20 & 0.20 & 0.20 & 0.20 & 0.20 \\
\hline \multicolumn{2}{|c|}{ Partial specific volume } & .10 & .05 & .02 & .11 & .06 & .02 \\
\hline \multicolumn{2}{|l|}{ Solute weights } & .19 & .14 & .38 & .18 & .15 & .36 \\
\hline \multicolumn{2}{|l|}{ Timer } & .19 & .19 & .20 & .13 & .14 & .15 \\
\hline \multicolumn{2}{|c|}{ Flow time correction factor $K$} & .28 & .28 & .28 & .08 & .08 & .08 \\
\hline \multicolumn{2}{|c|}{ Measurement temperature } & .10 & .10 & .10 & .10 & .10 & .10 \\
\hline \multicolumn{2}{|c|}{ Root-sum-square of the above } & .46 & .43 & .56 & .34 & .32 & .46 \\
\hline \multicolumn{2}{|c|}{$\begin{array}{l}\text { Sum of the individual error } \\
\text { estimates }\end{array}$} & 1.06 & .96 & 1.18 & .80 & .73 & .91 \\
\hline \multicolumn{2}{|c|}{$\begin{array}{l}\text { Expected limit of systematic } \\
\text { errors from all sources, in- } \\
\text { cluding sources not iden- } \\
\text { tified and treated here }\end{array}$} & 1 & 1 & 1 & 1 & 1 & 1 \\
\hline
\end{tabular}
be obtained directly.

TABLE 2. Percent errors in limiting viscosity numbers introduced by measured quantities

In the following subsections, the effects of errors in solution concentration and density, errors in measured flow time, and errors in $K$ are discussed in sections 4.1, 4.2, and 4.3 , respectively. Since $\Lambda$ is in general temperature-dependent, errors in the measurement temperature will give rise to implicit errors in $\Lambda$; these are discussed in section 4.4. Finally, the error limits from all sources are combined and summarized in section 4.5. 


\subsection{Errors in Solution Concentration and Density}

The effects of errors in solution density resulting from errors in solvent density and partial specific volume are shown by eq (11). We believe that for both solvents, our measurements of $\rho(0)$ are accurate to 0.2 percent, and that our values of $\bar{v}$ are accurate to about 3 percent, or $0.04 \mathrm{~mL} / \mathrm{g}$. By eq (11), a 0.2 percent relative error in $\rho(0)$ will lead to a 0.2 percent relative error in $\Lambda$, and an absolute error of $0.04 \mathrm{~mL} / \mathrm{g}$ in $\bar{v}$ will lead to an absolute error of the same size in $\Lambda$, leading to the relative errors in $\Lambda$ shown in table 2 .

The most direct source of possible systematic errors in solution concentrations is the balances used to weigh solute and solvent. Solute weights for SRM 1482 and solvent weights for all three SRM's were measured on semimicrobalances accurate to $0.1 \mathrm{mg}$. Solute weights for SRM 1483 and SRM 1484 were measured on an electrobalance accurate to $0.04 \mathrm{mg}$. The resulting errors in solvent weights are of the order of a few parts per million, and can be neglected. In order to estimate the effect of errors in the solute weights on the values of $\Lambda$, a series of comparison calculations was carried out. For each SRM and each solvent, a reference subset of typical data points was chosen, one at each concentration measured, and a "reference" value of $\Lambda$ was calculated from this set of points. The value for each solute weight in turn was then increased by its assumed limit of error, and the value of $\Lambda$ recalculated.The resulting percent changes in the reference values of $\Lambda$ are shown in table 3 , together with the sum of the absolute values of the individual changes, their algebraic sum, and the square root of the sum of their squares (root-sum-square). The sum of the absolute values represents the error in the case where every weighing is in error by the maximum amount possible and in the direction which maximizes the resulting error in $\Lambda$. We reject this estimate as overly pessimistic. The algebraic sum would be the appropriate measure if all the weighings were in error by the same amount, and the root-sum-square would be appropriate if the individual errors were of random sign. Since both these possibilities seem physically plausible, we select as our error estimate the larger of the absolute value of the algebraic sum and the root-sum-square. This turns out to be the absolute value of the algebraic sum for all three SRM's in TCB and for SRM 1482 in ICN, and the root-sum-square for SRM's 1483 and 1484 in ICN. The corresponding values are shown in table 2 .

A second possible source of systematic error in solution concentrations would arise if buoyancy corrections were not applied to solute and solvent weighings. Buoyancy corrections were applied to all measured solute and solvent weighings for SRM 1484, but not for SRM's 1482 and 1483. From the definition of weight fraction and the usual expression for the buoyancy correction, it is easily seen that as the concentration tends to zero, the correction factor $f(c)$ by which the "apparent" weight fraction (i.e., with uncorrected weighings) should be multiplied to give the true weight fraction approaches the limiting value

$$
f(0)=\left(1-\rho_{a} / \rho_{v}\right) /\left(1-\rho_{a} / \rho_{u}\right),
$$

where $\rho_{a}, \rho_{u}$, and $\rho_{v}$ are the densities of air, solute, and solvent, respectively. It is clear from the form of the limit expression in eq (11) that the limit is converted to its "corrected" value by dividing it by $f(0)$. Although this correction amounts to only about 0.03 percent for polyethylene in TCB and ICN, it affects the last digit in the values of $\Lambda$ given in table 1 , and was therefore applied to the data for SRM's 1482 and 1483.

\subsection{Timer Errors}

Viscometer flow times are measured to $0.001 \mathrm{~s}$. Spot checking suggests that they are accurate to at least $0.01 \mathrm{~s}$. The errors in $\Lambda$ resulting from assumed errors in flow time of $0.01 \mathrm{~s}$ were obtained by the same kind of comparison calculation described in 4.1 for errors in solute weight; the results are shown in table 4. Again, we reject the sum of the absolute values of the individual changes as being too pessimistic, and choose the larger of the absolute value of the algebraic sum of the individual changes and their rootsum-square. Since the viscosity number is essentially measured by the difference between solvent and solution flow times, it is not surprising that the algebraic sum of the individual changes including solvent is nearly zero. We therefore choose the root-sum-square as our error estimate, as shown in table 2 .

TABLE 3. Percent errors in limiting viscosity number $\Lambda$ introduced by assumed errors in solute weights

\begin{tabular}{|c|c|c|c|}
\hline Material & SRM 1482 & SRM 1483 & SRM 1484 \\
\hline $\begin{array}{l}\text { Measurements in TCB: Percent changes in } \\
\Lambda \text { from assumed errors in solute weight }\end{array}$ & $\begin{array}{l}-.04,-.07 \\
-.09,-.07 \\
+.08\end{array}$ & $\begin{array}{l}-.02,-.07 \\
-.08,-.04 \\
+.07\end{array}$ & $\begin{array}{l}-.01,-.20 \\
-.17,-.14 \\
+.14\end{array}$ \\
\hline Sum of absolute values of above & 0.35 & 0.26 & 0.66 \\
\hline Algebraic sum of above & -0.19 & -0.14 & -0.38 \\
\hline Root-sum-square of above & 0.16 & 0.13 & 0.33 \\
\hline $\begin{array}{l}\text { Measurements in ICN: Percent changes in } \\
\Lambda \text { from assumed errors in solute weight }\end{array}$ & $\begin{array}{l}-.03,-.06 \\
-.10,-.08 \\
+.09\end{array}$ & $\begin{array}{l}+.004,-.08, \\
-.10,-.03, \\
+.07\end{array}$ & $\begin{array}{l}+.03,-.17 \\
-.19,-.19, \\
+.17\end{array}$ \\
\hline Sum of absolute values of above & 0.36 & 0.28 & 0.75 \\
\hline Algebraic sum of above & -0.18 & -0.14 & -0.35 \\
\hline Root-sum-square of above & 0.17 & 0.15 & 0.36 \\
\hline
\end{tabular}


TABLE 4. Percent errors in limiting viscosity number $\Lambda$ introduced by assumed errors in measured flow times

\begin{tabular}{|c|c|c|c|}
\hline Material & SRM 1482 & SRM 1483 & SRM 1484 \\
\hline $\begin{array}{l}\text { Measurements in TCB: Percent changes in } \\
\Lambda \text { from assumed changes in: Solution } \\
\text { flow times }\end{array}$ & $\begin{array}{l}+.03,+.05 \\
+.06,+.05 \\
-.05\end{array}$ & $\begin{array}{l}+.02,+.07 \\
+.07,+.03 \\
-.06\end{array}$ & $\begin{array}{l}+.01,+.08, \\
+.06,+.05 \\
-.04\end{array}$ \\
\hline Solvent flow time & -.16 & -.15 & -.16 \\
\hline Sum of absolute values of above & 0.40 & 0.40 & 0.40 \\
\hline Algebraic sum of above & -0.02 & -0.02 & 0.00 \\
\hline Root-sum-square of above & 0.19 & 0.19 & 0.20 \\
\hline $\begin{array}{l}\text { Measurements in ICN: Percent changes in } \\
\Lambda \text { from assumed changes in: Solution } \\
\text { flow times }\end{array}$ & $\begin{array}{l}+.02,+.03 \\
+.05,+.04 \\
-.04\end{array}$ & $\begin{array}{l}-.002,+.05 \\
+.065,+.018 \\
-.04\end{array}$ & $\begin{array}{l}-.01,+.05 \\
+.05,+.05 \\
-.04\end{array}$ \\
\hline Solvent flow time & -.10 & -.10 & -.11 \\
\hline Sum of absolute values of above & 0.28 & 0.28 & 0.31 \\
\hline Algebraic sum of above & 0.00 & -0.01 & -0.01 \\
\hline Root-sum-square of above & 0.13 & 0.14 & 0.15 \\
\hline
\end{tabular}

\subsection{Errors in $\mathrm{K}$}

As described in section 3.1 , the correction factor $K$ was determined from the flow times $t_{N}$ and $t_{S}$ of two calibrating liquids of known viscosity and density. Equation (5) gives $K$ in terms of $t_{N}, t_{S}$, and the ratio $r$ of the viscosities and densities of the calibrating liquids. The ratio $r$ is equivalent to the ratio of flow times measured in special viscometers used for calibration. We believe that $r$ is accurate to 0.1 percent. As stated in section 3.1, 95 percent confidence limits of 0.02 percent and 0.03 percent can be assigned to our measured values of $t_{N}$ and $t_{S}$, respectively. Using the numerical values for these quantities in eq (5), we find that changes of these amounts in $r, t_{N}$ and $t_{S}$ produce changes in $K$ of 10 percent, 2 percent, and 3 percent respectively. The root-sum-square of the three error limits is 11 percent in $K$. The products $K t_{m}(0)^{-3}$ which occur in eq (11) are equal to 0.0088 for TCB and 0.0025 for $1 \mathrm{CN}$. From eq (11), errors of 11 percent in these products result in errors in $\Lambda$ of 0.28 percent and 0.08 percent for measurements in TCB and ICN, respectively, shown in table 2 .

\subsection{Errors in Measurement Temperature}

It is clear from eq (11) that so long as we have

$$
K t_{m}(0)^{-3}<<1
$$

and $\bar{v} \ll \Lambda$, the temperature dependence of $\Lambda$ will be essentially that of the product of $\rho(0)^{-1}$ and the limit

$$
\mathscr{L}\left\{\left[t_{m}(c)-t_{m}(0)\right] /\left[w t_{m}(0)\right\} .\right.
$$

The decrease in solvent density with temperature is roughly 0.1 percent per ${ }^{\circ} \mathrm{C}$ for both TCB and $1 \mathrm{CN}$, which would lead to an increase in $\Lambda$ of about the same size. Estimates of the temperature dependence of the limit quantity are not available. However, Chiang [8] has reported a decrease in $\Lambda$ of 0.2 percent per ${ }^{\circ} \mathrm{C}$ for linear polyethylenes in six theta solvents, consisting of aromatic hydrocarbons, ethers, and alcohols, and Flory, Ciferri and Chiang [9] report a decrease of 0.1 percent per ${ }^{\circ} \mathrm{C}$ for linear polyethylenes in three $n$ alkanes. Since the temperature dependence of $\rho(0)$ will always contribute an increase in $\Lambda$ with increasing temperature, typically of about 0.1 percent per ${ }^{\circ} \mathrm{C}$, it appears that the limit quantity must decrease with temperature at two or three times this rate. Lacking more directly applicable data, we choose 1 percent per ${ }^{\circ} \mathrm{C}$ as a safe upper limit for the change in $\Lambda$ with temperature in the solvents employed in this work. We believe that our measurements of the viscometer bath temperature are accurate to $0.1^{\circ} \mathrm{C}$. The corresponding uncertainty in $\Lambda$ of 0.1 percent is shown in table 2 .

\subsection{Summary}

Estimates of the contributions of individual sources to the possible systematic errors in $\Lambda$ are summarized in table 2 , together with their sums and root-sum-squares. As previously discussed, we believe that the sum of the absolute values of the individual errors is an unduly pessimistic estimate of the error from all the sources discussed here, and that the rootsum-squares constitute a more reasonable estimate. To take account of sources of error not considered explicitly here, we round each of the root-sum-square estimates, which range from 0.32 percent to 0.56 percent, upward to 1 percent in all cases. Finally, we note that our final error estimates of 1 percent are exceeded by the sum of the absolute values of individual error estimates in only two out of the six cases, and that the largest sum of absolute values, for SRM 1484 in TCB, is only 1.18 percent. We therefore believe that our arbitrary limits of 1 percent for systematic errors from all sources are quite likely to be realistic. These estimates are those given on the certificates for SRM's 1482, 1483, and 1484 .

\section{References}

[1] Verdier, P. H., and Wagner, H. L., The characterization of linear polyethylene SRM's 1482, 1483, and 1484. I. Introduction, J. Res. Nat. Bur. Stand. (U.S.) 83, No. 2, (Mar.-Apr. 1978). 
[2] Wagner, H. L., and Hoeve, C. A. J., Mark-Houwink relations for linear polyethylene in 1-chloronaphthalene and 1,2,4-trichlorobenzene, J. Polymer Sci. (Polymer Physics Ed.) 11, 1189-1200 (1973).

[3] See, for example, Yamakawa, H., Modern Theory of Polymer Solutions Ch. VI (Harper \& Row, 1971).

[4] See, for example, Hardy, R. C., NBS viscometer calibrating liquids and capillary tube viscometers, Nat. Bur. Stand. (U.S.), Monogr. 55, 22 pages (Dec. 1962)

[5] Cannon, M. R., Manning, R. E., and Bell, J. D., Viscosity measurement: the kinetic energy correction and a new viscometer, Anal. Chem. 32, 35.5-358 (1960).

[6] See, for example, Statistical Methods in Research and Production,
Davies, O. L. Ed. (Hafner Publishing Co., New York, 1961); Bennett, C. A. and Franklin, N. L., Statistical Analysis in Chemistry and the Chemical Industry (John Wiley \& Sons, Inc., New York, 1954).

[7] See, for example, Onyon, P. F., Viscometry, Ch. 6 in Techniques of Polymer Characterization, P. W. Allen, Ed. (Butterworths Publications Ltd, London, 1959).

[8] Chiang, R., Temperature coefficient of the unperturbed dimension of linear polyethylene from intrinsic viscosity measurements in $\theta$ solvents, J. Phys. Chem. 70, 2348-2352 (1966).

[9] Flory, P. J., Ciferri, A., and Chiang, R., Temperature coefficient of the polyethylene chain conformation from intrinsic viscosity measurements, J. Am. Chem. Soc. 83, 1023-1026 (1961). 